\title{
INFODEMIA E GOVERNO ALGORÍTMICO: AS NOVAS TECNOLOGIAS DE BOTS AFETAM O POPULISMO CONTEMPORÂNEO BRASILEIRO?
}

\author{
INFODEMIC AND ALGORITHMIC GOVERNMENT: DO THE NEW BOT \\ TECHNOLOGIES AFFECT THE CONTEMPORARY BRAZILIAN POPULISM?
}

Recebimento em 21/09/2020

Aceito em 28/09/2020

\author{
Rafael Beltrão Urtiga \\ Carina Barbosa Gouvêa
}

\section{RESUMO}

A pesquisa apresenta a concepção teórica da infodemia em razão da pandemia da COVID-19 com aplicação do uso de tecnologia de botscom foco na governamentabilidade algorítmica e populismos. Terá como objetivo apresentar suas principais características para que se possa posteriormente proceder a sua materialização em um processo de know-how. O caminho percorrido para a sua concretude baseou-se na metodologia hipotética-dedutiva com métodos advindos de fontes bibliográficas nacionais e estrangeiras, com foco na fenomenologia.

Palavras-chave: Infodemia. COVID-19. Governo Algorítmico. Populismo. Brasil.

\section{ABSTRACT}

The research out lines the theoretical concept of infodemia due to the COVID-19 pandemic in regards with the application of the use of bot technology focus in gon both algorithmic governance and populism. Also, It aims at presenting its main characteristics so as to proceed its manifest under a know-how process. To achieve the objective, a hypothetical-deductive methodology from nation a landforeign sources has been required, focused on phenomenology.

Keywords: Infodemic. COVID-19. Algorithmic Government. Populism. Brazil.

\section{INTRODUÇÃO}

O título da pesquisa, de certo, num primeiro momento, pretende alertar sobre os fenômenos tecnológicos compreendidos dentro de uma óptica de governo algorítmico que afeta sistemas políticos, a democracia e o Brasil. Infodemia, para alguns, gera certa surpresa em razão do desconhecimento relativo à temática. Para outros, certa convicção ao confirmar uma possível 
indicação científica de uma percepção empírica. E, por fim, para os demais, traz sensação de indiferença, dada a ausência de reflexão sobre o tema.

De toda forma, antes de analisar os institutos de Infodemia, Populismos e Bots, é necessário traçar alguns propósitos que norteiam a pesquisa: a busca pelo apoio popular é o sustentáculo de um governo democrático, pois todas as suas medidas, no âmbito interno ou externo, apoiam-se, ainda que indiretamente, na reação do povo a qual se destinam ou representam; a governança populista ${ }^{1}$ se utiliza, dentre outras variáveis ${ }^{2}$, do enfraquecimento e ataques às salvaguardas institucionais; da utilização maciça dos serviços de inteligência e de controle de dados e vigilância, da polarização da política sectária por líderes carismáticos; da rotinização do carisma; dos discursos retóricos e demagogos como particulares estilos de comunicação extremista; do comportamento antidemocrático; do uso retórico de políticas e ações governamentais; da exploração do sentimento popular de descrédito nas instituições; da política mítica; do uso sistemático do ambiente midiático em plataformas sociais como WhatsApp, Twitter, Facebook; do uso e impulsionamento desenfreado de desinformações e fake new, dentre outros comportamentos que se enquadrem nos princípios antidemocráticos e iliberais (GOUVÊA; CASTELO BRANCO, 2020), no prelo. Estas variáveis ${ }^{3}$ se decantam através dos movimentos formais e informais de forma direta ou indireta no campo do iliberalismo democrático (GOUVÊA; CASTELO BRANCO, 2020).

Neste cenário, não só é importante compreender o papel desempenhado pelas redes sociais no jogo político, uma vez que, a concentração do uso da internet em plataformas de redes sociais tem o condão de acentuar opiniões e consensos polarizados de forma a acelerar a

\footnotetext{
${ }^{1}$ A governança populista, para Issacharoff, representa uma forma de corrupção da governança democrática pela ação populista que reflete a corrupção da própria soberania popular. Pretende o populista, através de ações políticas, a curto prazo, de forma imediata, sem preocupação com o futuro, recompensar e favorecer um universo clientelista. Ver: ISSACHAROFF, Samuel. The Corruptionof Popular Sovereignty. ICON, N. 20-02, 2020, NYU Schoolof Law, Public Law ResearchPaper No. 20-02, NYU LawandEconomicsResearchPaper No. 20-02. (GOUVÊA, Carina Barbosa; CASTELO BRANCO, Pedro H. Villas Bôas. Populismos. 2020. No prelo.)

${ }^{2}$ Estratégias comumente utilizadas em contextos políticos específicos com o intuito de ampliar a força política do movimento. (GOUVÊA, Carina Barbosa; CASTELO BRANCO, Pedro H. Villas Bôas. Populismos. 2020. No prelo.)

${ }^{3}$ Estas variáveis foram extraídas da vasta literatura que gira no entorno do populismo e não constituem um rol terminativo. Para maiores informações consulte as referências.
} 
manifestação de posicionamentos que antes eram mediados por pessoas em diferentes contextos de maneira cadenciada (MACHADO; MISKOLCI, 2019).

Assim sendo, tem-se que com a virada tecnológica dos últimos anos, a externalização da opinião popular migrou massivamente para a rede mundial de computadores: a internet. Atualmente, as redes sociais ocupam posição de protagonismo no campo político e jurídico,vez que permitem às pessoas defenderem interesses, estabelecerem críticas às iniciativas políticas e sugerirem pautas de interesse metaindividual.

Diante disso, é possível imaginar que qualquer representante governamentaldemocrático e com base no Estado de Direito preocupa-se com a opinião pública que se encontra conectada à rede, principalmente em momentos de crises, tal como a emergência em saúde pública global decorrente do surto da COVID-19.

A preocupação do representante do governo com a opinião popular, além de servir como um termômetro de aceitação das medidas implementadas, também permite ampliar o espaço de vocalização e diálogo com a comunidade para a proposição de outras medidas. Todavia, duas hipóteses são essenciais: Como lidar com ferramentas tecnológicas capazes de comprometer a neutralidade da rede? Como confiar em informações disparadas em massas por uma única fonte?

Vivemos uma pandemia. Desde o dia 11 de março de 2020, a Organização Mundial de Saúde - OMS, em razão do surto de Coronavirusdisease (COVID-19), determinou adoção de protocolo de emergência com recomendações de distanciamento social.

No Brasil, apesar de controvertida a temática, a Suprema Corte Brasileira ${ }^{4}$ reforçou a competência concorrente da União, dos Estados e dos Municípios para enfrentamento da pandemia, afastando qualquer interpretação que pudesse restringir "a tomada de providências normativas e administrativas pelos Estados, Distrito Federal e Municípios", especialmente sobre circulação de mercadorias e pessoas, reforçando a ideia de federalismo centrífugo ou cooperativo. A situação é crítica, não só pelos níveis alarmantes de óbitos e infectados pela COVID-19, mas também pela dificuldade de gerenciamento de informações que circulam sobre a doença no país.

A pandemia decorrente da COVID-19 representa um cenário de caos que afetará todas as gerações, como ocorreu com aqueles indivíduos que vivenciaram a situação pandêmica da gripe

\footnotetext{
${ }^{4}$ Ainda que monocraticamente, decidiu o Supremo Tribunal Federal na ADI n. ${ }^{\circ} 6341$ pela ratificação do Pacto Federativo, estabelecendo como concorrente a competência dos Estados e Municípios, sem prejuízo ou a despeito da União para lidar com questões de saúde. Posteriormente, a decisão liminar foi ratificada, declarando que o exercício de competência federal sobre normas de proteção à saúde não exclui a dos Estados e Municípios de estipularem diversamente, observado o disposto no art. 198, I, da Constituição Federal, já que, em tal matéria, a competência é concorrente.
} 
espanhola $^{5}$. Para o contexto atual, há isolamento, distanciamento, do convívio social ao mesmo tempo que revela uma hiperconexão seja pela internet, telefone ou televisão.

Apesar do contato físico ter sido drasticamente reduzido e o prognóstico ser de recessão econômica, todos os dias novas ferramentas tecnológicas surgem e, independente da sua finalidade - seja terapêutica, de recreação ou vigilância, coletam dados, tornando-nos ainda mais conectados. Nesta linha,sobressalta o devastador número de contágios e mortes ocorridas pela pandemia do coronavírus. Em estimativa recente, pesquisadores da Johns Hopkins University (2020) contabilizaram a marca histórica de mais 7 milhões de pessoas infectadas pelo COVID19, bem como totalizaram mais de 400 mil mortes nos últimos meses. No contexto brasileiro, o último boletim divulgado, também pela Johns Hopkins University (2020), são mais de 670 mil pessoas infectadas e 40 mil óbitos.

De certo, em todo o mundo, o cenário foge da valoração atípica e encaminha-se para crítica. Em decorrência disso, diversos governos, nos mais diversos continentes, viabilizaram iniciativas, quer sejam isoladas, quer sejam conjuntas, para buscar a minimização da propagação do vírus.

No âmbito nacional, inúmeros governos, após decretarem estado de exceção em virtude da deflagração do estado de emergência em saúde pública global, viabilizaram iniciativas tecnológicas para buscar precipuamente a redução do número de contágios, seja por meio da identificação de locais de maior probabilidade de contaminação ou pela imposição metaindividual de isolamento. Estas iniciativas buscam, precipuamente, a redução do número de contágios.

Todavia, essas iniciativas tendem-se a colidir com uma variedade de direitos humanos, dentre eles: privacidade e segurança de dados. Importante destacar que não é de agora a existência da problemática, entretanto, à medida que a inovação tecnológica caminha a passos largos, impõe o inevitável sopesamento entre direitos individuais e promoção do bem coletivo.

O desafio atual no cenário jurídico, portanto, é entender o alcance das normas protetivas de direitos e garantias individuais e a limitação do poder estatal frente à adoção de medidas de restrição. Em outras palavras, reavivou-se o antigo debate dos fins justificando os meios, da ética em confronto com a moral, e, principalmente, da transparência nas escolhas.

\footnotetext{
${ }^{5}$ Estimam os historiadores, sem muita precisão científica, que no mínimo ocorreram 50 milhões de mortes, entre os anos de 1918 e 1920 , em todo o mundo por causa da gripe espanhola.
} 


\section{DA NECESSÁRIA ADEQUAÇÃO DOS CONCEITOS: DESINFORMAÇÃO, FAKE NEWS E INFODEMIA.}

Segundo a OMS (2020), as informações difundidas na internet, mais precisamente nas redes sociais, vinculam expressivo quantitativo de informações sobre a pandemia, sendomuitas dessas informações com qualidade e credibilidade comprometida, ensejando no fenômeno da desinformação.

Na literatura especializada (GUESS; NYHAN; REIFLER, 2018), já se reconhece como um novo tipo específico de desinformação a questão política, marcada por uma "dubiedade factual com finalidade lucrativa" instrumentalizadas especificamente em campanhas eleitorais, principalmente, para os altos cargos do Poder Executivo. Importante mencionar que o fenômeno da desinformação possui ligação direta com a circulação de fakenews, e embora não seja este o foco do trabalho, pode ser compreendida em duas modalidades: identificáveis, cuja a essência se aproxima de sátiras, com habitual valor humorístico; e nãoidentificáveis, que possui lastro factual duvidoso, porém, de certa forma convincente, capaz de levantar suposta veracidade quanto ao conteúdo (AYMANNS; FOERSTER; GEORG, 2017).

Nessa linha, as fakenews, em sua modalidade não identificável, acabam por contribuir para o fenômeno da desinformação, que por sua vez, entrelaça-se com a problemática da pesquisa: infodemia.

Para a OMS (2020), a desinformação não se confunde com o conceito de infodemia. Isto porque a desinformação possui natureza de imprecisão, seja pela falta de base científica ou, não raro, pela falta de verdade. Por outro lado, ainfodemia representa o grande fluxo de informações circuladas sobre o COVID-19, que enseja em sérios riscos à saúde global.

O recorte metodológico aqui pretendido refere-se especificamente com a liberdade de comunicação baseada na transmissão de fatos e notícias. Tal pressuposto é relevante vez que,se tratando do exercício de liberdade comunicativa, de cunho individual, não haveria necessidade de comprovação da verdade interna da noção transmitida, pois crenças e opiniões podem ser criticadas e desacreditadas sem que se possa avaliar o seu grau de verdade para o agente, o que não ocorreria com a liberdade de comunicação baseada na transmissão de fatos e notícias (GRINGS, 2019).

De certo, em termos objetivos, especificamente voltado para uma aplicação prática dos conceitos, não se tem de forma cartesiana a divisão do que seria uma liberdade de comunicação baseada na transmissão de fatos e notícias do que seria liberdade comunicativa de cunho individual (FARIAS, 2004). 
Compreende-se como governo algorítmico o uso dos algoritmos pela gestão de governamentalidade de qualquer figura estatal. Este tipo de governabilidade algorítmica utilizase da lógica de ferramentas tecnológicas para direcionar a ação de indivíduos, estruturando as interações entre o humano e a máquina em velocidade instantânea, com adisponibilização do máximo de informações sobre os interesses e necessidades dos seus usuários (TELES, 2018). Consiste na externalização de uma normatividade decorrente do deslocamento e circulação dos dados capaz de fornecer curadoria específica de experiências sociais e políticas com mitigação dos instrumentos comuns de debates. Em outras palavras, trata-se de interferência direta na formação do senso coletivo.

\section{POPULISMOS}

Apresentados os conceitos que norteiam a pesquisa, apresenta-se, desde logo, a sua relação com o contexto brasileiro, especificamente no que diz respeito a onda contemporânea de populismo.

Apesar do reconhecimento do grande léxico semântico da expressão do populismo e, por conseguinte, da dificuldade de um estabelecimento de conceito preciso e sólido, opta-se aqui por uma abordagem de know-how, elencando características essenciais para sua identificação.

Para Gouvêa e Castelo Branco (2020), a materialização do fenômeno populista é identificada a partir das suas propriedades variáveis, que devem ser compreendidas em contextos culturais e políticos específicos. Para tanto, os autoresempregam o uso da expressão não no singular, considerando mais adequada a sua representação no plural: populismos.

Com o propósito de extrair do leque semântico a inflexão de seus sentidos, Gouvêa e Castelo Branco (2020) consideram que populismos têm natureza conceitual-instrumental e podem ser definidos como iliberalismo democrático com propriedades variáveis. Representam um certo tipo de ideologia, discurso, estratégia, mobilização e ação política no contexto cultural e político específico. Se decanta através de movimentos formais e/ou informais de forma direta ou indireta no sistema democrático por um líder carismático que representa e conduz uma força antistablishmentrepousando suas crenças em instituições morais e éticas com a finalidade de consolidar e legitimar um regime político populista sob o manto da soberania popular e da democracia.

Os populismos se decantam no sistema democrático liberal com o intuito de degradar de forma estrutural as democracias constitucionais neoliberais. Pretende se materializar como uma forma de governo específica, distinta do totalitarismo e autoritarismo e que, pressupõe a ascensão 
de um líder carismático que não se diz político, mas sim um salvador do estado de crise, propondo-se a reformular as práticas de governanças existentes através de movimentospopulistas diretos e indiretos. Estes movimentos são percebidos através de propriedades variáveis, como, por exemplo, o uso sistemático do ambiente midiático em plataformas sociais como WhatsApp, Twitter, Facebook; do uso e impulsionamento desenfreado de desinformações e fakenews; do comportamento antidemocrático, dentre outros (GOUVÊA; CASTELO BRANCO, 2020).

\section{DAS NOVAS TECNOLOGIAS DE BOTS}

Botsconsistem em software simples, que empregam ferramentas mínimas de inteligência artificial e habilidades comunicativas textuais para apresentar uma informação de maneira automatizada. Atualmente, elessão projetados com propósitos específicos, mas com finalidade comum: fornecer um serviço mais rápido e conciso, sem compelir as pessoas a dedicarem longas horas para obter aquilo que pretendem (TEIXEIRA, 2018).

No que discerne a tipologia das ferramentas de bots, sãocategorizadas em simples (keywords) e complexas (machinelearning). Os botsdo tipo simples (keywords)consistem em um sistema capaz de responder a frases pré-formuladas, substituir tarefas extensas e trabalhosas com metodologia simples de dialógo entre o usuário e a plataforma, atendo aos propósitos idealizados pelo seu disponiblizador, podendo, inclusive ser integrado ou não a um sistema.

Por sua vez, as ferramentas de botscomplexas (machinelearning), possuem os mesmos elementos da simples (keywords), só que ganham também condições de aprendizagem com análises constantes das palavras trocadas ao longo do tempo, levando-se em consdieração a frequência de apariação e etc., permitindo a sua categorização como uma inteligência artificial (TEIXEIRA, 2018).

Em resumo, as ferramentas de bots, independente de serem dos tipos simples ou complexos, se estruturam de forma tríplice: 1) receptor - (responder) é a parte que desempenha o papel da interface entre o bot e o utilizador; 2) classificador - (classifier) etapaonde se filtra e normaliza a entrada de dados, segmentando-os de maneira lógica; 3 ) executor - (graphmaster) fase em que se executa as tarefas em correspondência com os padrões (STONER; FORD; RICCI, 2004). Vejamos:

Figura 1 - Infográfico sobre o funcionamento de Bots. 


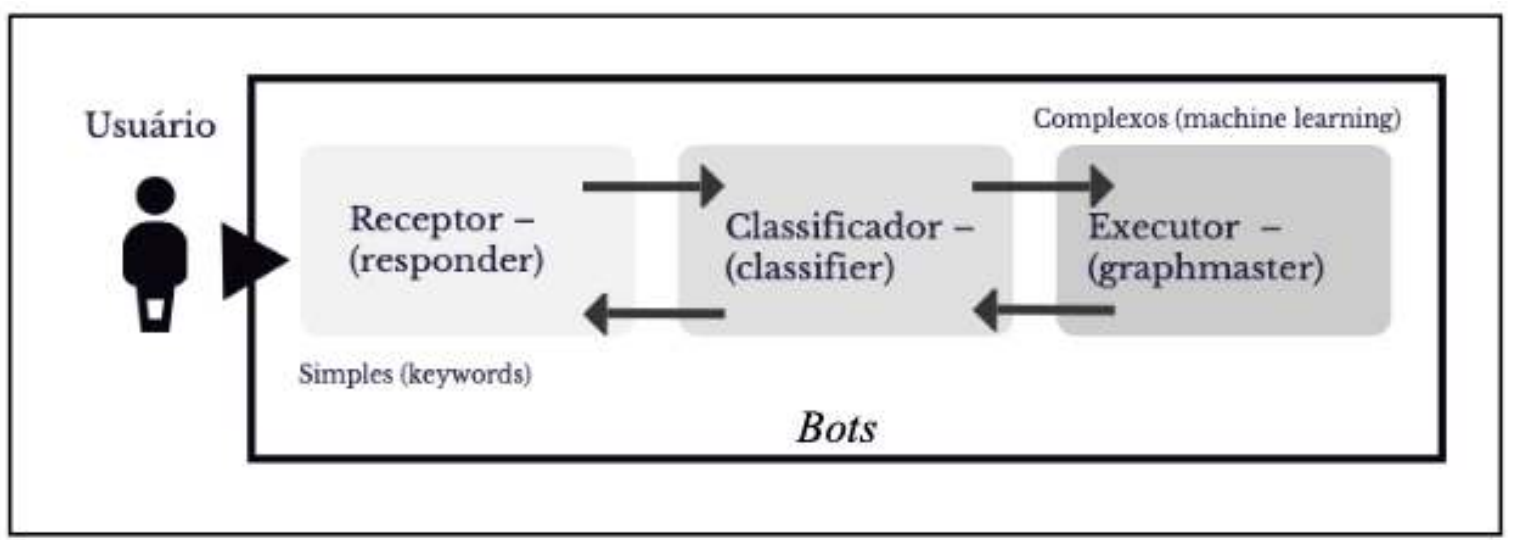

Fonte: Os autores.

Sua recorrente aplicação, e, por conseguinte, a ratificação de sua utilização, cresceu exponencialmente nos últimos anos, tornando-se acessível a diversos públicos que vão desde vão desde serviços de atendimento ao cliente em instituições bancárias (TEIXEIRA, 2018) até protótipos para o auxílio do tratamento de doenças crônicas (OLIVEIRA; COSTA; ARAUJO; PORTELA, 2019). Sua popularização se deve ao fato de existirem plataformas de desenvolvimento rápido - Chatfuel, o Botsify, etc. -que ajudam na criação de bots sem ser necessário possuir grandes aptidões de programação (TEIXEIRA, 2018).

Estas plataformas permitem que se adicione funcionalidades ao bot, como integração para outras interfaces de programação de aplicações (APIs), tal como as redes sociais do FacebookMessenger, WhatsApp, por exemplo, estabelecendo um fluxo de conversação ou condições de aprendizagem não concentrada no diálogo, mas nas respostas por intermédio das escolhas, no qual baseia-se em um fluxograma preestabelecido, ou seja, o layout já definido pelo programador, cabendo ao usuário apenas escolher dentre as opções as possíveis aplicações desejadas (TEIXEIRA, 2018).

Embora esse seja o uso mais comum, os fins comerciais, a pesquisa se centra em abordar o emprego de ferramentas de botsnas redes sociais, com ênfase na projeção de opinião, sejam as ligadas a difusão de fatos e notícias, ou ainda, na projeção da liberdade comunicativa de cunho individual que, por permitir uma veloz massificação dos discursos, torna-se uma ferramenta capaz de comprometer a credibilidade do espaço e da informação.

\subsection{DA ADEQUAÇÃO DOS CONCEITOS DE SUJEITOS E CONDUTA: BOTS, TROLLS,} BOTNETS E TROLLING

Estabelece-se como premissa neste tópico a análise da conduta incompatível do usuário com determinado termo de uso e política de privacidade de comunidade estabelecida no âmbito virtual. Sendo assim, tomando como certo que qualquer usuário ao entrar em uma comunidade 
virtual (rede social) concorda com suas condições e políticas de privacidade e, portanto, tem o dever de cumprir todos os ditames normativos de condutas para que se enquadre como usuário. Porém, há usuários com condutas incompatíveis às políticas da comunidade virtual onde se destacam três categorias: bots, trolls e botnet. Épossível que estes usuários que infringem as regras e políticas determinadas, também pratiquem trolling.

Especificamente sobre os bots, conforme já explicitado em parágrafos anteriores, trata-se de um sistema projetado para realizar atividade de forma automatizada, fazendo-se ou não se passar por uma pessoa real. Por outro lado, os trollssão usuários reais, não automatizados por bots, com condutas incompatíveis aos termos de uso e as políticas de privacidade, que se utiliza de uma comunicação violenta de forma intencionalpara implantar conflitos, ofender usuários e causar dissensões contrárias às regras preestabelecidas.

Os botnetssão uma coletividade de contas de bots gerenciadas pelo mesmo núcleo. Importante destacar que, o núcleo responsável por botnets, antes da implantação, são reconhecidos como geradores - "botherders" ou "botshepherds" - capazes de fabricar engajamento de mídia social, fazendo com que a temática difundida aparente maior relevância em razão de ter sido fomentada por supostamente usuários reais, já que não se tem como claro para os integrantes da rede quem seria botsou não (BAROJAN, 2018).

Ainda sobre os botnetstem-se que sua formulação depende de três componentes básicos os bots, os servidores de comando e controle (C\&C) e o núcleo, constituindo uma infraestrutura de medição multifacetada e distribuída e representando um grande contribuidor para o tráfego indesejado da Internet, cerca de $27 \%$ de todas as tentativas de conexão maliciosas (RAJAB, ZARFOSS, MONROSE, TERZIS, 2006).

Para melhor compreensão, vejamos:

Figura 2 - Infográfico sobre o funcionamento dos botnets. 


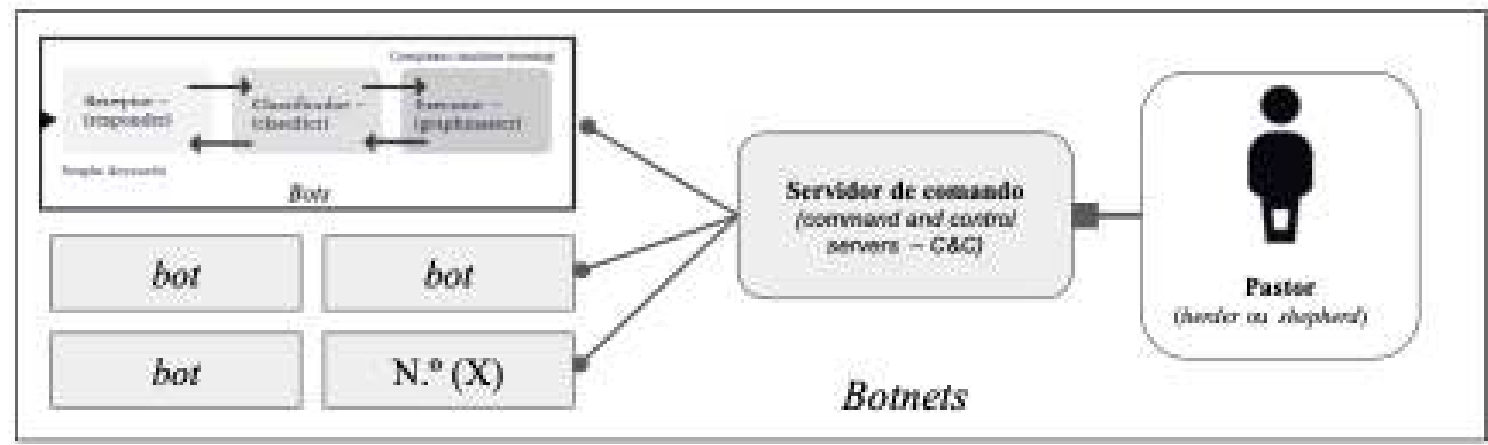

Fonte: Os autores.

De certo, tanto a internet, aqui compreendida como um aspecto geral, como as redes sociais, compreendidas como um aspecto específico, tornaram-se um importante espaço para debate político. Fatores com dinamismo e facilidade de acesso abrem um espaço para discussões legítimas e factuais, quer sejam fulcrados na liberdade comunicativa individual ou na transmissão de fatos e notícias.

Sendo assim, diante deste próspero ambiente para a disseminação de opiniões, a automatização de ferramentas de publicação possibilitou o surgimento e a propagação por meio de bots. Nesse contexto, temos o estudo Diretoria de Análise de Políticas Públicas, da Fundação Getúlio Vargas, que identificou a recorrência de contas controladas por softwares se fazendo passar por seres humanos (FGV/DAPP, 2017). A pesquisa ainda indica, em razão de interações periódicas, que tais contas tidas como falsas (fakes), por serem contas controladas por bots se fazendo passar por seres humanos, são capazes de participar ativamente das discussões em momentos políticos de grande repercussão (FGV/DAPP, 2017).

A materialização dessa participação ativa ocorre por meio de botsquevão desde promover determinado tipo de conteúdo e/ou pessoa, conquistando seguidores e, por conseguinte, possíveis apoiadores, bem como também a conduzir ataques determinados, forjar discussões artificiais, manipular debates e replicar mensagens em larga escala a fim de promover hashtags por meio de massificação de postagens automatizadas, ensejando numa sufocação de algum debate espontâneo sobre algum tema (FGV/DAPP, 2017).

E não é só, atualmente, apesar de várias discussões junto a jurisdição eleitoral ${ }^{6}$, também é relevante a problemática do uso de bots para promoção de mensagens de apoio às medidastomadas pelo Presidente da República no Brasil. A pesquisa desenvolvida

\footnotetext{
${ }^{6}$ Tramita junto ao Tribunal Superior Eleitoral a Ação de Investigação Judicial Eleitoral (Aije) $n^{\circ}$ 0601782-57, que investiga o uso de disparo em massa de mensagens via WhatsAppdurante as eleições para presidência no ano de 2008. Afastados os juízos de valores, tem-se como fundamentos jurídicos da ação de investigação o 'abuso de poder econômico' e 'uso indevido dos meios de comunicação', em afronta ao art. 14, § $9^{\circ}$, da CRFB/88, e art. 22, da Lei no 64/90.
} 
pelaUniversidade Federal do Rio de Janeiro (UFRJ) em parceria com a Fundação Escola de Sociologia e Política de São Paulo (FespSP), realizada em 2020, analisou pormenorizadamente todas as postagens de apoio a gestão do governo Bolsonaro realizadas em redes sociais nos últimos meses.

\subsection{DA VERIFICAÇÃO DO FENÔMENO: RECORTE METODOLÓGICO E PROBLEMÁTICA ESTRUTURANTE}

Nesse contexto, dentre os muitos eventos que poderiam ser analisados, em razão do alto tráfego de bots em redes sociais sincronizado a momentos políticos específicos, um em especial merece destaque: As novas tecnologias de bots constituem uma variável de populismo no atual governo brasileiro? O evento ocorrido em 15 de março de 2020 permite constatar que o tráfego de postagens realizadas por não humanos, ou melhor, por botsse fazendo passar por humanos, de acordo com os dados públicos o compartilhamento de mensagens no Twitter no Brasil atingiu expressiva recorrência com o levantamento deHASHTAGS ${ }^{7}$ de apoio do Poder Executivo Federal.

Especificamente sobre as mensagens de apoio, tratavam-se de enaltecimento pela eficiência de tratamento com hidroxicloroquina, remédio amplamente usado no combate a doenças como lúpus e artrite reumatoide (DANZA; GRAÑA; GOÑI, RUIZ-IRASTORZA, 2016), mas sem comprovação de sua eficácia e segurança contra a COVID-19 (FALAVIGNA, 2020).

As hashtags $^{7}$ tiveram aproximadamente 1 milhão e duzentas mil menções alcançando os trendingtopics. Após estudos posteriores constatou-se que os dados circulados formaram uma ação expressiva de perfis não humanos nas postagens do Twitter(KALIL; SANTINI, 2020). A pesquisa concluiu que em eventos específicos, em especial um ocorrido em 15 de março, 55\% dos 1,2 milhão de posts realizados em homenagem ao Executivo foram inseridos na internet por meio de bots(KALIL; SANTINI, 2020). Por meio da técnica de etnografia também sustentaram as pesquisadoras Isabela Kalil e Marie Santini (2020) que a disseminação de conteúdo em massa, em apoio ao governo de Bolsonaro, não foi restrito a uma única rede social, mas sim a diversas ${ }^{8} \mathrm{e}$

\footnotetext{
${ }^{7}$ Em linhas gerais, hashtagssão tags usadas em redes sociais para marcar publicações de conteúdo similares, de forma a proporcionar maior visibilidade do tema; a sua função é similar a de palavras chaves em produções científicas.
} 
foram utilizadas especificamente como termômetro para averiguação da opinião pública sobre temáticas ligadas a COVID-19.

Diante das problemáticas apresentadas, tem-se como expressiva ao alerta com relação a neutralidade da rede e a governabilidade algorítmica que acabam por potencializar a desinformação e contribui significativamente para o fenômeno da infodemia $e$ de populismos.

No campo da governabilidade algorítmica, não deve haver confusão entre a atuação de botscom trolls. Em suma, os trollsrepresentam contas controladas por seres humanos, ou seja, trata-se de um ser humano, que intencionalmente suscita conflito na redeprejudicando a interação com outros usuários para subverter espaço de determinada rede social. Em outras palavras, o trollé um usuário com padrão incompatível ao termos de uso e política de privacidade. Sendo assim, temos que o troll se diferencia do bot por ser usuário real, a contrassenso de uma estrutura automatizada, mas que ambos são capazes de comprometer a neutralidade da rede, bem como implementar o aspecto negativo de governo algorítmicos em uma atividade essencialmente prejudicial, reconhecida como trolling.

\section{CONCLUSÃO}

A pesquisa apresentou duas hipóteses: Como lidar com ferramentas tecnológicas capazes de comprometer a neutralidade da rede? Como confiar em informações disparadas em massas por uma única fonte? Foi possível concluir que o uso de bots e trollspotencializam o campo da governança populista e algorítmica.

A proposição apresentada para possível minimização das ações a criação de um protocolo de responsabilidade e transparência (accountabilityandtransparency) que deve ser necessariamente aplicado a toda rede que difunde informações, principalmente as de conteúdo essencialmente político ${ }^{8}$.

A pesquisa não abordousobre a moralidade do emprego de botsnas redes sociais para difusão de conteúdo. Pelo contrário, o que se questionou no presente estudo é a sua utilização em anonimato, por intermédio de botnets, que por decorrência macula a formação do senso comum. É importante destacar que o usuário não possui conhecimento técnico paraverificar se a informação foi difundida por bot, de maneira automatizada, ou por um usuário semelhante, o que notadamente prejudica o ideal de transparência.

\footnotetext{
${ }^{8}$ De certo, a expressão “de qualquer conteúdo"possui viés político, pois em suma representa a defesa de uma ideia, seja por meio de criação, reformulação ou reprodução. Todavia, a tônica dada à expressão 'essencialmente político' se refere a questão política partidária, de forma a ratificar ou discordar de determinada medida adotada.
} 
Por fim, sobre a confiabilidade de informações disparadas em massa por uma única fonte, tem-se que essa ação prejudica a participação democrática, não só porque a busca pelo apoio popular é o sustentáculo de um governo democrático, mas também por permitir que populismos possam se naturalizar através da cooptação das massas populares agravando as crises já decantadas no sistema democrático.

A pesquisa ainda conclui que a participação popular na rede deva ocorrer por dupla forma: em caráter decisório, preceituando à abertura de canais para que o particular, em sua individualidade, participe ativamente do processo de formação das políticas públicas, de forma a influenciar constantemente o processo decisório do seu governo; e, em caráter de cooperação, que envolve a ideia de que o poder executivo não é autossuficiente, e, portanto, demanda a integração e colaboração da sociedade civil e demais poderes para alcançar os fundamentos da sociedade política e normativa que a que está integrado.

\section{REFERÊNCIAS}

ARGUELHES, Diego Werneck. Symposium: theBrazilianSupremeCourtandtheProtectionofDemocracy in the Age ofPopulism: The SupremeCourtandthe Bolsonaro Government: A FragmentedCourt in a ConflictivePoliticalScenario, Int'l J. Const. L. Blog, jun. 2019.

AYMANNS, C.; FOERSTER, J.; GEORG, C.P. Fake News in Social Networks. ArXivpreprint arXiv:1708.06233.

BAROJAN, Donara. Noções básicas sobre bots, botnets e trolls. Digital ForensicResearchLab do AtlanticCouncil (DFRLab), nov. 2018.

CONTINENTINO, Marcelo Casseb; MEDICIS, Ernani Varjal. Estamos diante de um novo federalismo brasileiro? Conjur,abr. 2020. Disponível em: https://bit.ly/2MZRS1r. Acesso em: 8 jun. 2020.

DANZA, Álvaro et al . Hidroxicloroquinaeneltratamiento de lasenfermedadesautoinmunes sistémicas. Rev. méd.Chile, Santiago, v. 144, n. 2, p. 232-240, feb. 2016.

FALAVIGNA, Maicon et al . Diretrizes para o tratamento farmacológico da COVID-19. Consenso da Associação de Medicina Intensiva Brasileira, da Sociedade Brasileira de Infectologia e da Sociedade Brasileira de Pneumologia e Tisiologia. Rev. bras. ter. intensiva, São Paulo, v. 32, n. 2, p. 166-196, jun. 2020.

FARIAS,Edilsom Pereira de. Liberdade de expressão e comunicação: teoria e proteção constitucional. São Paulo: Revista dos Tribunais, 2004. 
FREITAS, Carolina. 55\% de publicações pró-Bolsonaro são feitas por robôs. Valor Econômico, São Paulo,abr. 2020. (Caderno de política). Disponível em: https://glo.bo/3hb591x. Acesso em: 7 jun. 2020.

GOUVÊA, Carina Barbosa; CASTELO BRANCO, Pedro H. Villas Bôas. Populismos, 2020.

GRINGS, Maria Gabriela. Publicidade processual, liberdade de expressão e super-injunction. São Paulo: Thomson Reuters Brasil, 2019.

GUESS, A.; NYHAN, B.;REIFLER, J. SelectiveExposuretoMisinformation: Evidencefromtheconsumptionoffakenewsduringthe 2016 US presidentialcampaign.

KALIL, I. \& SANTINI, R. M. Coronavírus, Pandemia, Infodemia e Política. Relatório de pesquisa. São Paulo: Rio de Janeiro: FESPSP / UFRJ, 2020. 21p. Disponível em: https://bit.ly/3cKAyYL. Acesso em:8 jun. 2020.

KEARNEY, Michael W. Tweetbotornot: DetectingTwitterbots. Researchgate, jun. 2018. Disponível em: https://bit.ly/30t1VUR. Acesso em: 8 jun. 2020.

MACHADO, Jorge; MISKOLCI, Richard. Das jornadas de junho à cruzada moral: o papel das redes sociais na polarização política brasileira. Sociol. Antropol., Rio de Janeiro , v. 9, n. 3, p. 945-970, dez. 2019. Disponível em:https://bit.ly/2V3C42j. Acesso em: 8 jun. 2020.

OLIVEIRA, Natália; COSTA, Allan; ARAUJO, Diovanni; PORTELA, Carlos. HelpCare: Um Protótipo de ChatBot para o Auxílio do Tratamento de Doenças Crônicas. In: SIMPÓSIO BRASILEIRO DE COMPUTAÇÃO APLICADA À SAÚDE (SBCAS), 19., 2019, Niterói. Anais [...]. Porto Alegre: Sociedade Brasileira de Computação, 2019. p. 282-287.

PAHO. Pan American Health Organization. Entenda a infodemia e a desinformação na luta contra a COVID-19. Departamento de Evidência e Inteligência para Ação em Saúde da Organização Mundial da Saúde (OMS), n. 5, abr. 2020. Disponível em:https://bit.ly/2YEv0K9. Acesso em: 8 jun. 2020.

PROCEEDINGSofthe 6th ACM SIGCOMM conferenceon Internet measurement, 2006.

RAJAB, Moheeb Abu; ZARFOSS, Jay; MONROSE, Fabian; TERZIS, Andreas. A multifaceted approach tounderstandingthebotnetphenomenon. IMC, 2006 ?

RUEDIGER, Marco Aurélio.Robôs, redes sociais e política no Brasil: estudo sobre interferênciasilegítimas no debate público na web, riscos à democracia e processo eleitoral de 2018. Rio de Janeiro : FGV, DAPP, 2017.

STONER, Donald J.; FORD, Louis; RICCI, Mark. Simulatingmilitary radio communications using speech recognitionand chat-bottechnology. The Titan Corporation, Orlando, 2004.

TEIXEIRA, Fábio André Alves.Chatbot para serviços bancários. Orientador: Alípio Mário Guedes Jorge. 2018. Dissertação (Mestrado Integrado em Engenharia de Redes e Sistemas 
Informáticos) - Departamento de Ciência de Computadores,Faculdade de Ciências, Universidade do Porto, Porto, 2018.

TELES, Edson. Governamentalidade Algorítmica e as Subjetivações Rarefeitas. Kriterion,Revista de Filosofia, Belo Horizonte, v. 59, n. 140, p. 429-448, ago. 2018. Disponível em: https://bit.ly/3dMqMqr. Acesso em:8 jun. 2020. 